\title{
Gliomatosis cerebri: quantitative proof of vessel recruitment by cooptation instead of angiogenesis
}

\author{
Hans Bernsen, M.D., Ph.D., Jeroen van der Laak, Ph.D., Benno Küsters, M.D., \\ Abel van der Ven, M.D., And Pieter Wesseling, M.D., Ph.D. \\ Department of Neurology, Canisius Wilhelmina Hospital; and Department of Pathology, \\ Nijmegen Centre for Molecular Life Sciences, Radboud University Nijmegen Medical Centre, \\ Nijmegen, The Netherlands
}

\begin{abstract}
Object. Gliomas are the most common primary brain tumors, many of which (especially astrocytic and oligodendroglial neoplasms) are characterized by diffuse infiltrative growth in the preexisting brain tissue. Gliomatosis cerebri is a rare glial tumor and represents an extreme example of such diffuse infiltrative growth. This growth pattern not only hampers curative treatment but also allows for vessel cooptation rather than tumor angiogenesis as a way of vessel recruitment by the tumor tissue. The goal of this study was to establish the extent to which tumor angiogenesis occurs in gliomatosis cerebri.

Methods. Computerized image analysis was performed to assess quantitatively two microvascular parameters (vessel density and diameter) in different areas of a brain harboring a gliomatosis cerebri. These regions were the cerebral white and gray matter in which there was a diffuse infiltrative tumor, cerebral white and gray matter in which there was a more compact growth pattern of tumor cells, and normal cerebral white and gray matter. In addition, the authors performed immunohistochemical stainings for blood-brain barrier (BBB) characteristics (Glut-1 and PgP) on samples obtained in these different areas.

The results of the quantitative analysis strongly indicated that in gliomatosis cerebri tumor, angiogenesis was completely absent, a finding that is corroborated by the fact that the microvasculature in gliomatosis cerebri persists in exhibiting immunohistochemical characteristics of the BBB.

Conclusions. The results of this study may help resolve the difficulties in radiological detection and delineation of the diffuse infiltrative part of glial brain tumors and put the expectations for antiangiogenic treatment of such tumors into perspective.
\end{abstract}

\section{KEY WordS • gliomatosis cerebri • vessel cooptation • angiogenesis • quantitative analysis}

$\mathrm{G}$ LIOMAS are the most common primary brain tumors. Many of these neoplasms, especially astrocytic and oligodendroglial lesions, are characterized by diffuse infiltrative growth in the preexisting brain tissue, accompanied by a more solid component of variable size and intensity ${ }^{32}$ The diffuse infiltrative growth pattern hampers not only radiological/neuroimaging detection and delineation but also topical curative treatment (for example, by surgery or irradiation) of these tumors. Gliomatosis cerebri is a rare neoplasm, defined by the WHO-2000 classification of tumors of the nervous system ${ }^{13}$ as a diffuse glial tumor infiltrating the brain extensively, involving more than two lobes, frequently bilaterally, and often extending to infratentorial structures. In some cases, even the entire neural axis might be involved. ${ }^{5}$ The prognosis in patients with gliomatosis cerebri is usually poor; according to the WHO-2000 classification, gliomatosis cerebri is considered to represent Grade III malignancy, although long-term survival has been reported in some individuals.

Clinically the presentation of gliomatosis cerebri varies

\footnotetext{
Abbreviations used in this paper: $\mathrm{BBB}=$ blood-brain barrier; $\mathrm{CT}=$ computerized tomography; $\mathrm{MR}=$ magnetic resonance; VEGF $=$ vascular endothelial growth factor; $\mathrm{WHO}=$ World Health Organization.
}

widely, ranging from symptoms mimicking vascular disease to mental illness or epileptic manifestations. ${ }^{1,28}$ Unfortunately CT and MR imaging characteristics are also nonspecific for this condition, thus making a clinical diagnosis difficult. Histopathologically, one often observes a diffuse and sometimes symmetrical cellular infiltration of glial cells in the preexisting brain tissue with relative preservation of the neuronal architecture.

That a gliomatosis cerebri does not usually exhibit enhancement on CT or MR imaging suggests that angiogenesis may not be one of its features. This is in contrast with the notion that most tumors, probably also including high-grade gliomas, require angiogenesis for their growth. ${ }^{8,23}$ To establish the role of neovasularization in gliomatosis cerebri, we performed computerized image analyses to assess vessel density and diameter in histological sections of multiple pathological and normal areas of a brain in which a gliomatosis cerebri was present. In addition, the presence of $\mathrm{BBB}$ characteristics was investigated in these areas by using immunohistochemical markers (Glut-1 and PgP). To our knowledge, this is the first quantitative study of the vascularity of gliomatosis cerebri. Analysis of our results indicates that angiogenesis was absent in the gliomatosis cerebri, suggesting that tumor growth in these neoplasms is supported by cooptation of the existing vasculature and not by the formation of new vessels. 


\section{Vessel cooptation in gliomatosis cerebri}
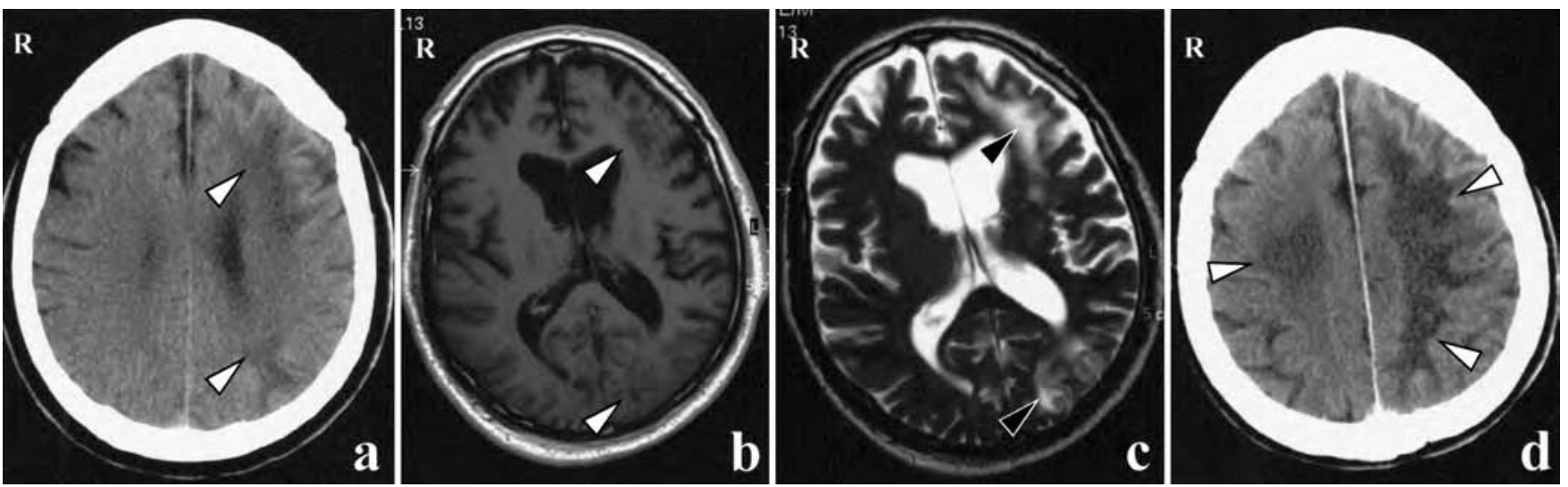

FIG. 1. a: Transverse CT scan demonstrating a large subcortical hypodense area in the left hemisphere on first admission to the hospital. $\quad \mathrm{b}$ and $\mathrm{c}$ : Transverse $\mathrm{T}_{1}$-weighted $\mathrm{Gd}$-enhanced and $\mathrm{T}_{2}$-weighted MR images revealing a large subcortical area in the left cerebral hemisphere that is hypointense but does not enhance after contrast administration (b), and exhibits increased signal on a $\mathrm{T}_{2}$-weighted sequence (c). d: Transverse CT scan obtained 4 months after initial admission, demonstrating increased hypodensity in not only the left but also the right cerebral hemisphere (again without contrast enhancement). Arrowheads indicate the pathological areas; $\mathrm{R}=$ frontal right side.

\section{Materials and Methods}

\section{Clinical Data}

This 68-year-old man presented to the hospital with a 1-month history of phatic disturbances and cognitive dysfunction. His family reported that his complaints began suddenly. His medical history was unremarkable except for a 3-year history of atrial fibrillation. Neurological examination revealed a Mini-Mental State score of 18, a regular pulse, mild right hemiparesis (including a central facial paresis), and mild disturbance in word fluency and comprehension. Transverse CT scanning revealed a large subcortical hypodense area in the left cerebral hemisphere (Fig. 1a). Transverse $\mathrm{T}_{2}$-weighted MR imaging demonstrated a large subcortical area of increased signal intensity in this hemisphere (Fig. 1b and c), but contrast enhancement was absent. Before the neurological workup could be finished, the patient was admitted to a nursing home and lost to follow up for 4 months. When he was readmitted to the hospital, the patient suffered from mutism and quadriparesis with increased, generalized muscle rigidity and bilateral impaired reflexes. Transverse CT scanning now revealed increased hypodensity of the white matter in the left cerebral hemisphere, as well as in the right hemisphere, still without contrast enhancement (Fig. 1d). A pulmonary infection developed and the patient died a few days after readmission. Autopsy examination (including the brain) indicated that pneumonia was the cause of death.

\section{Neuropathological Findings}

The brain weight (1410 g) was within normal range. Attached to the dura mater, a small parasagittal meningioma (maximal diameter $1.5 \mathrm{~cm}$ ) was found in the parietooccipital region. The brain showed some signs of swelling (moderate flattening of cerebral gyri, as well as moderate prominence of parahippocampal gyri and cerebellar tonsils), but no evidence of herniation. The brain swelling might have been caused by hypoxic damage in an agonal phase rather than by the brain tumor, especially because CT scans obtained several days prior to death revealed no brain swelling. After formalin fixation, the brain was cut into $1-\mathrm{cm}$-thick slices. On the cut surface, the white matter in the left frontal, parietal, and occipital lobes showed widespread, poorly demarcated softening with dispersed rarefaction. In the basal cortex of the left occipital lobe, we observed a more circumscribed, small lesion (maximum dimension $1 \mathrm{~cm}$ ) exhibiting granular texture. Multiple samples were acquired of both cerebral hemispheres, the cerebellum, and the brainstem, and embedded in paraffin; 4- $\mu \mathrm{m}$ histological sections were cut and stained with $\mathrm{H} \&$ E and a combined Luxol fast blue-H \& E staining. Microscopically, the more circumscribed occipital lobe lesion consisted of densely packed, relatively small, elongated cells with oval or elongated nuclei, moderate nuclear pleomorphism, little eosinophilic cytoplasm, and focally high mitotic activity. In the periphery of this lesion, perivascular cuffs of a highly cellular tumor were found. In the left frontal, parietal, and occipital lobes, as well as in the right half of the corpus callosum, extremely extensive, diffuse infiltrative growth of tumor cells was found in both gray and white matter, accompanied by rarefaction of the preexisting white matter, some reactive astrocytosis, and occasional macrophages. Necrosis and florid microvascular proliferation were absent. In line with the definition of the WHO-2000 classification, ${ }^{13}$ the tumor in this case was diagnosed as a gliomatosis cerebri.

\section{Quantitative Analysis}

Quantitative analysis of the the microvasculature in histologically normal and pathological brain tissue obtained in this patient was performed as previously described. ${ }^{33,34}$ In short, histological sections of paraffin-embedded tissue samples were immunohistochemically stained with a monoclonal anti-collagen IV antibody (Sigma Chemical Co., St. Louis, MO), resulting in clear-cut demarcation of the intracerebral microvasculature. Computerized quantitative analysis was performed using the VidasPlus system (Kontron $\mathrm{GmbH}$, Eching, Germany). Microscopic fields measuring $400 \times 425 \mu \mathrm{m}$ were selected from the following areas of the cerebrum for further microvasculature quantitative analysis: normal neocortex (number of analyzed microscopic fields, 15); normal white matter (number of microscopic fields, 30); neocortex with diffuse infiltrative tumor from the left frontal lobe (convex and basal areas) and from the basal part of the occipital lobe (number of microscopic fields, 43); white matter with diffuse infiltrative tumor from the left frontal, left occipital, and right frontal lobes (number of microscopic fields, 97); neocortex with compact tumor from the basal area of the left occipital lobe (number of microscopic fields, 28); and white matter with compact tumor from the basal area of the left occipital lobe (number of microscopic fields, eight). In each microscopic field the number of separate vascular profiles was assessed. Additionally, the diameter of each individual vascular profile was measured. Statistical evaluation was performed using SPSS 10.0 for Windows (SPSS, Inc., Chicago, IL). A one-way analysis of variance was used to study the differences among the aforementioned areas. Because the Levene test showed an inhomogeneity of variances $(\mathrm{p}<0.001)$, Tamhane post hoc tests were used in the analysis of variance. Finally, histological sections of paraffin-embedded tissue samples were immunohistochemically stained for BBB markers with the monoclonal antibodies 

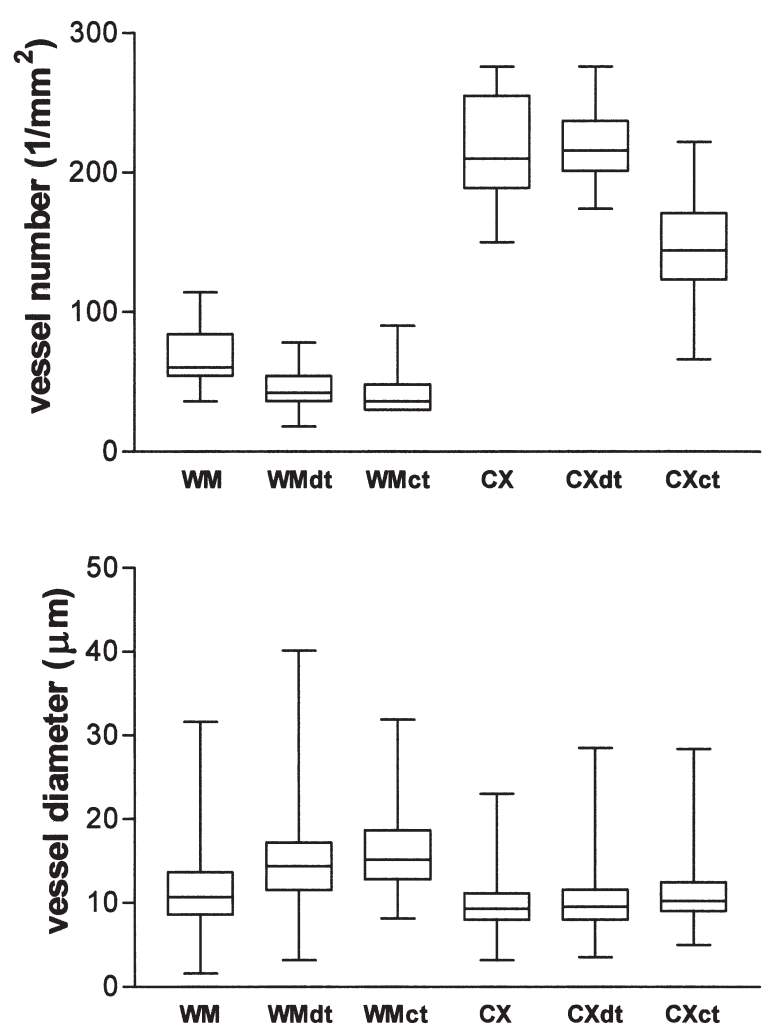

FIG. 2. Box plots representing median (transverse line in box), 75th, and 25th percentile (upper and lower end of boxes) and highest and lowest values (upper and lower end of lines) for vessel number and diameter in the following cerebral areas: normal white matter (WM), white matter with diffuse infiltrative tumor (WMdt), white matter with compact tumor (WMct), normal neocortex (CX), neocortex with diffuse infiltrative tumor (CXdt), and neocortex with compact tumor (CXct). Note that in both white matter and cortex, the presence of diffuse or compact tumor is associated with a decrease, rather than increase, in vessel number, whereas the vessel diameter tends to be somewhat higher in tumor-infiltrated areas.

Glut-1 and PgP (Dako, Glostrup, Denmark) after standard antigen retrieval (citrate buffer and microwave) as previously described. ${ }^{16}$

\section{Results}

The vessel number in tumor-infiltrated white matter and cortex was equal to or significantly lower than in their normal brain counterparts (Fig. 2). The vessel diameter in tumor-infiltrated areas tended to be somewhat higher than in normal brain tissue, but this difference did not reach statistical significance. The results of the quantitative analysis are depicted in Fig. 2. Examples of the microvascular parameters in different tumor areas are shown in Fig. 3. In both normal and tumor-infiltrated cerebral cortex and white matter, almost all microvessels stained positively for the BBB markers Glut-1 and PgP (Fig. 4).

\section{Discussion}

The role of angiogenesis in the growth and malignant progression of tumors in general, ${ }^{8}$ and in malignant gliomas in particular, has gained considerable attention in the last

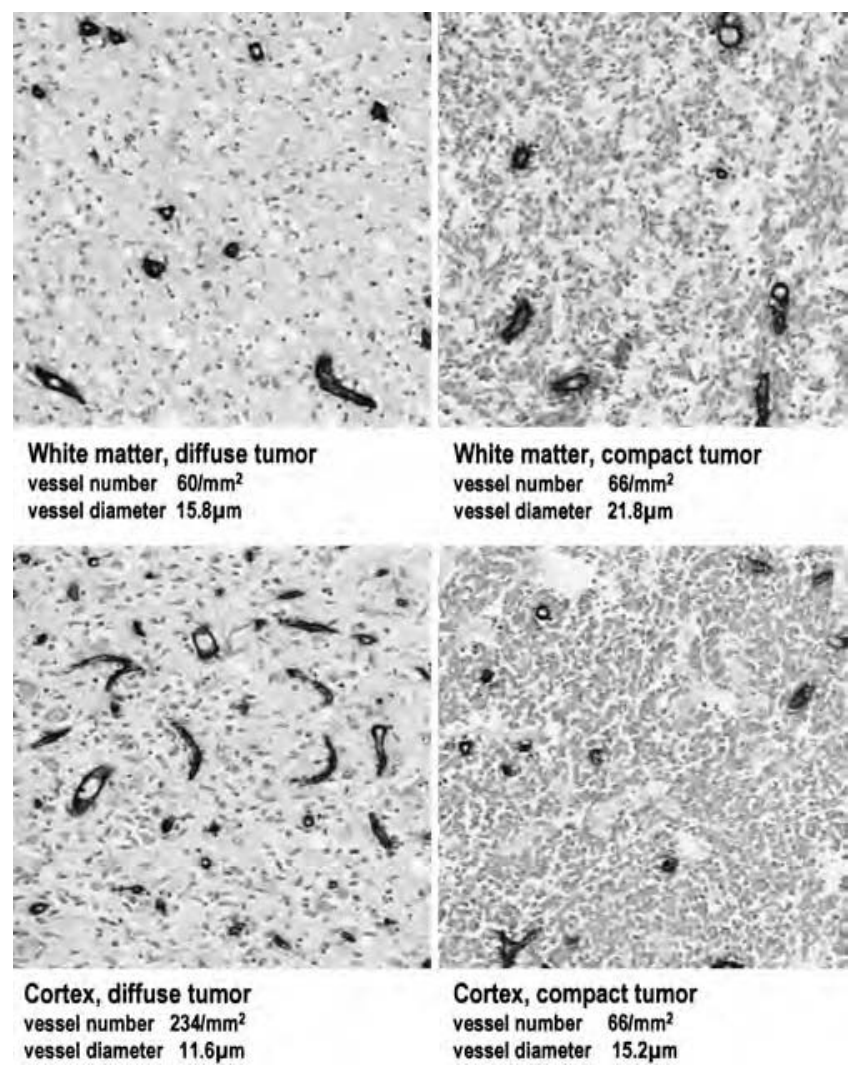

FIG. 3. Photomicrographs providing examples of quantitative analysis of microvascular parameters in cerebral white matter and cortex with diffuse infliltrative or more compact tumor growth. The images show the immunohistochemical staining for collagen Type IV, which highlights the basement membrane of the microvasculature and which was used for computerized image analysis. Original magnification $\times 100$.

decade. ${ }^{23}$ Endothelial/microvascular proliferation and contrast enhancement as signs of neovascularization, however, are rarely mentioned in conjunction with gliomatosis cerebri. Gliomatosis cerebri is an entity known for its extremely diffuse infiltration of neoplastic cells in the brain tissue, often with minimal destruction of preexisting structures. ${ }^{3,9}$, ${ }^{13,30}$ In fact, such diffuse infiltrative growth may very well allow the tumor to recruit vessels by cooptation rather than angiogenesis. The main purpose of our examination was to evaluate the extent of angiogenesis in gliomatosis cerebri. To our knowledge, this is the first report in which a systematic study of the vascularization in gliomatosis cerebri is presented.

Our quantitative vascular analysis confirmed that, in contrast to other high-grade gliomas, ${ }^{34}$ in our patient with gliomatosis cerebri, neovascularization was not a characteristic feature. This finding indicates that tumor progression was not facilitated by the development of a neovascular bed; rather, the mechanism of migration and infiltration into the preexisting brain parenchyma allowed tumor growth. This phenomenon has recently been described as "coöption" of the preexisting vascular bed. ${ }^{18,25}$ Interestingly, it was not only described in the brain parenchyma ${ }^{11,14-17,21,26}$ but also in the skin, ${ }^{6}$ lung, ${ }^{20,22}$ and liver ${ }^{29,31}$

The presence of vessel cooptation, the absence of neovas- 


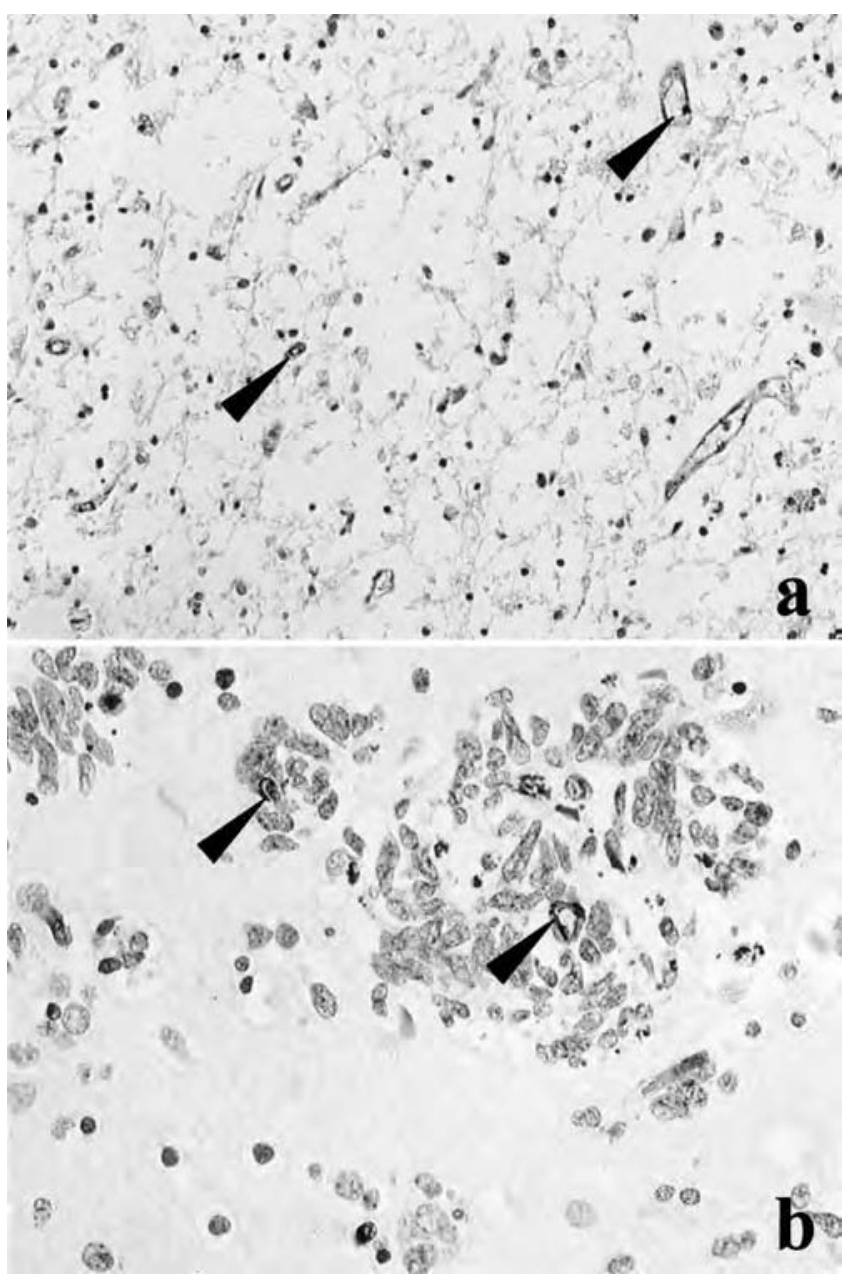

FIG. 4. Photomicrographs showing the BBB markers PgP (a) and Glut-1 (b) in cerebral white matter with diffuse infiltrative (a) and cortex with perivascular, more compact tumor (b). Note the staining of the microvessels (arrowheads), strongly indicating that these are preexisting, incorporated vessels rather than newly formed vessels. Original magnification $\times 100$ (a) and $\times 200$ (b).

cularization, and the apparently limited changes to the preexisting, incorporated vessels in gliomatosis cerebri explain why these lesions do not enhance on MR images after contrast administration..$^{35}$ Because both clinical symptoms and neuroradiological findings are often nonspecific, histological examination is generally required to establish the nature of this condition. ${ }^{12}$ Often considered are demyelinating diseases, inflammatory or infectious processes (for example, progressive multifocal leukencephalopathy), and ischemic changes or leukodystrophia. ${ }^{24}$

Vascular leakage and neovascularization are VEGF-dependent processes. ${ }^{7}$ In a mouse model with intracerebral human melanoma xenografts, we recently showed that even using high-resolution (7-tesla) MR imaging, VEGF-negative brain metastases are hardly detectable. ${ }^{17}$ Histologically, such tumors grow by cooptation of the preexisting vasculature. ${ }^{15,16}$ When such tumors were engineered to produce VEGF, however, they were easily demonstrated on MR images as contrast-enhancing tumors. ${ }^{15,17}$ On MR imaging, anaplastic astrocytoma and glioblastoma multiforme typi- cally exhibit areas of contrast enhancement, which correspond to areas of vascular changes (vasodilation or glomeruloid microvascular proliferation) at the histological level. Such changes have been shown to be VEGF dependent. ${ }^{10}$ Interestingly, in experimental glioblastoma multiforme models, the blockade of vascular changes by anti-VEGF therapy resulted in increased vessel cooptation by the tumor. ${ }^{14,26}$ In our murine model of intracerebral human melanoma metastases, we also recently demonstrated that VEGF blockade leads to a cooptative phenotype and makes their MR imaging detection difficult. ${ }^{19}$

To date, the treatment of gliomatosis cerebri usually consists of external radiotherapy and corticosteroid therapy.,12 Radiation therapy may be useful in some patients with gliomatosis cerebri, ${ }^{4}$ although the duration of effects is limited. ${ }^{2}$ Recently, Sanson and colleague ${ }^{27}$ reported an effect after temozolomide and procarbazine-lomustine-vincristine chemotherapy, especially in patients with slow-growing gliomatosis cerebri.

\section{Conclusions}

Based on our present and previous findings we conclude that, although MR imaging-documented contrast enhancement of parts of brain tumors may be used as an argument for responsiveness to antiangiogenic therapy, gliomatosis cerebri and the nonenhancing portion of other diffuse infiltrative gliomas are very unlikely to respond to such therapy.

\section{References}

1. Artigas J, Cervos-Navarro J, Iglesias JR, Ebhardt G: Gliomatosis cerebri: clinical and histological findings. Clin Neuropathol 4: 135-148, 1985

2. Choi D, Schulz U, Seex K: Gliomatosis cerebri: a brain tumour which is too difficult to treat? Scott Med J 43:84-86, 1998

3. Couch JR, Weiss SA: Gliomatosis cerebri. Report of four cases and review of the literature. Neurology 24:504-511, 1974

4. Cozad SC, Townsend P, Morantz RA, Jenny AB, Kepes JJ, Smalley SR: Gliomatosis cerebri. Results with radiation therapy. Cancer 78:1789-1793, 1996

5. Cummings TJ, Hulette CM, Longee DC, Bottom KS, McLendon RE, Chu CT: Gliomatosis cerebri: cytologic and autopsy findings in a case involving the entire neuraxis. Clin Neuropathol 18: 190-197, 1999

6. Dome B, Paku S, Somlai B, Timar J: Vascularization of cutaneous melanoma involves vessel co-option and has clinical significance. J Pathol 197:355-362, 2002

7. Ferrara N: VEGF and the quest for tumor angiogenesis factors. Nat Rev Cancer 2:795-803, 2002

8. Folkman J: What is the evidence that tumors are angiogenesis dependent? J Natl Cancer Inst 82:4-6, 1990

9. Freund M, Hahnel S, Sommer C, Martmann M, Kiessling M, Tronnier V, et al: CT and MRI findings in gliomatosis cerebri: a neuroradiologic and neuropathologic review of diffuse infiltrating brain neoplasms. Eur Radiol 11:309-316, 2001

10. Goldbrunner RH, Bendszus M, Sasaki M, Kraemer T, Plate KH, Roosen $\mathrm{K}$, et al: Vascular endothelial growth factor-driven glioma growth and vascularization in an orthotopic rat model monitored by magnetic resonance imaging. Neurosurgery 47:921-930, 2000

11. Holash J, Maisonpierre PC, Compton D, Boland P, Alexander $\mathrm{CR}$, Zagzag D, et al: Vessel cooption, regression, and growth in tumors mediated by angiopoietins and VEGF. Science 284: 1994-1998, 1999 


\section{H. Bernsen, et al.}

12. Kim DG, Yang HJ, Park IA, Chi JG, Jung HW, Han DH, et al: Gliomatosis cerebri: clinical features, treatment, and prognosis. Acta Neurochir (Wien) 140:755-762, 1998

13. Kleihues P, Cavanee WK: Pathology and Genetics of Tumours of the Nervous System. Lyon: IARC Press, 2000

14. Kunkel P, Ulbricht U, Bohlen P, Brockmann MA, Fillbrandt R, Stavrou D, et al: Inhibition of glioma angiogenesis and growth in vivo by systemic treatment with a monoclonal antibody against vascular endothelial growth factor receptor-2. Cancer Res 61: 6624-6628, 2001

15. Kusters B, de Waal RM, Wesseling P, Verrijp K, Maass C, Heerschap A, et al: Differential effects of vascular endothelial growth factor $\mathrm{A}$ isoforms in a mouse brain metastasis model of human melanoma. Cancer Res 63:5408-5413, 2003

16. Kusters B, Leenders WP, Wesseling P, Smits D, Verrijp K, Ruiter DJ, et al: Vascular Endothelial Growth Factor-A(165) induces progression of melanoma brain metastases without induction of sprouting angiogenesis. Cancer Res 62:341-345, 2002

17. Leenders W, Kusters B, Pikkemaat J, Wesseling P, Ruiter D, Heerschap A, et al: Vascular endothelial growth factor-A determines detectability of experimental melanoma brain metastasis in GD-DTPA-enhanced MRI. Int J Cancer 105:437-443, 2003

18. Leenders WP, Kusters B, de Waal RM: Vessel co-option: how tumors obtain blood supply in the absence of sprouting angiogenesis. Endothelium 9:83-87, 2002

19. Leenders WP, Kusters B, Verrijp K, Maass C, Wesseling P, Heerschap A, et al: Anti-angiogenic therapy of cerebral melanoma metastases results in sustained tumor progression via vessel co-option. Clin Cancer Res 10:6222-6230, 2004

20. Passalidou E, Trivella M, Singh N, Ferguson M, Hu J, Cesario A, et al: Vascular phenotype in angiogenic and non-angiogenic lung non-small cell carcinomas. Br J Cancer 86:244-249, 2002

21. Peoch M, Farion R, Hiou A, Le Bas JF, Pasquier B, Remy C: Immunohistochemical study of VEGF, angiopoietin 2 and their receptors in the neovascularization following microinjection of C6 glioma cells into rat brain. Anticancer Res 22:2147-2151, 2002

22. Pezzella F, Pastorino U, Tagliabue E, Andreola S, Sozzi G, Gasparini G, et al: Non-small-cell lung carcinoma tumor growth without morphological evidence of neo-angiogenesis. Am J Pathol 151:1417-1423, 1997

23. Plate KH, Risau W: Angiogenesis in malignant gliomas. Glia 15: 339-347, 1995

24. Ponce P, Alvarez-Santullano MV, Otermin E, Santana MA, Garcia Ludena MV: Gliomatosis cerebri: findings with computed tomography and magnetic resonance imaging. Eur J Radiol 28: 226-229, 1998

25. Ribatti D, Vacca A, Dammacco F: New non-angiogenesis depen- dent pathways for tumour growth. Eur J Cancer 39:1835-1841, 2003

26. Rubenstein JL, Kim J, Ozawa T, Zhang M, Westphal M, Deen $\mathrm{DF}$, et al: Anti-VEGF antibody treatment of glioblastoma prolongs survival but results in increased vascular coöption. Neoplasia 2:306-314, 2000

27. Sanson M, Cartalat-Carel S, Tallibert S, Napolitano M, Djafari L, Cougnard J, et al: Initial chemotherapy in gliomatosis cerebri. Neurology 63:270-275, 2004

28. Schoenen J, De Leval L, Reznik M: Gliomatosis cerebri: clinical, radiological and pathological report of a case with a stroke-like onset. Acta Neurol Belg 96:294-300, 1996

29. Stessels F, van den Eynden G, van der Auwera I, Salgado R, van den Heuvel E, Harris AL, et al: Breast adenocarcinoma liver metastases, in contrast to colorectal cancer liver metastases, display a non-angiogenic growth pattern that preserves the stroma and lacks hypoxia. Br J Cancer 90:1429-1436, 2004

30. Vates GE, Chang S, Lamborn KR, Prados M, Berger MS: Gliomatosis cerebri: a review of 22 cases. Neurosurgery 53:261-271, 2003

31. Vermeulen PB, Colpaert C, Salgado R, Royers R, Hellemans H, van den Heuvel E, et al: Liver metastases from colorectal adenocarcinomas grow in three patterns with different angiogenesis and desmoplasia. J Pathol 195:336-342, 2001

32. Wesseling P, Ruiter DJ, Burger PC: Angiogenesis in brain tumors: pathobiological and clinical aspects. J Neurooncol 32:253-265, 1997

33. Wesseling P, van der Laak JA, de Leeuw H, Ruiter DJ, Burger PC: Quantitative immunohistological analysis of the microvasculature in untreated human glioblastoma multiforme. Computer-assisted image analysis of whole-tumor sections. J Neurosurg 81: 902-909, 1994

34. Wesseling P, van der Laak JA, Link M, Teepen HL, Ruiter DJ: Quantitative analysis of microvascular changes in diffuse astrocytic neoplasms with increasing grade of malignancy. Hum Pathol 29:352-358, 1998

35. Yang S, Wetzel S, Law M, Zagzag D, Cha S: Dynamic contrastenhanced $\mathrm{T} 2 *$-weighted MR imaging of gliomatosis cerebri. AJNR Am J Neuroradiol 23:350-355, 2002

Manuscript received November 10, 2004.

Accepted in final form May 2, 2005.

Dr. Wesseling received support (Grant No. KUN2003-2975) from the KWF Kankerbestrijding/Dutch Cancer Society.

Address reprint requests to: Hans Bernsen, M.D., Ph.D., Department of Neurology, Canisius Wilhelmina Hospital, P.O. Box 9015, 6500 GS Nijmegen, The Netherlands. email: h.bernsen@cwz.nl. 